\title{
Interfacial Instabilities of Shear-Thinning Fluids in Homogeneous Porous Media
}

\author{
Y.-H. Lee, J. Azaiez, I. D. Gates \\ Department of Chemical and Petroleum Engineering \\ Schulich School of Engineering \\ University of Calgary, Calgary, Canada, T2N 1N4
}

\begin{abstract}
In this study, radial immiscible flow displacements in homogeneous porous media are modelled numerically in the case where the displacing fluid is Newtonian while the displaced one may be Newtonian or shear-thinning governed by Carreau's rheological model. The governing equations and interfacial jump conditions are solved numerically using the Immersed Interface Method and the Level Set Method to track the two fluids interfaces. The effects of four parameters, namely the mobility ratio and Capillary number for Newtonian flows and the Deborah number $D e$ and power-law index $n$, for shear thinning fluids are examined. Time contours of the interface revealing the finger structures that develop as a result of the viscosity mismatch between the two fluids are obtained to analyse and discuss the effects of the different parameters on the interfacial instability. Even though both characteristics of the shear-thinning fluid; $D e$ and $n$ were found to affect the interfacial instability, it is revealed that the latter has a stronger effect, at least for the range of parameters that have been considered in this study.
\end{abstract}

Keywords: Viscous Fingering, Immiscible Flow, Non-Newtonian Flow, Porous Media, Numerical Simulations.

\section{Introduction}

Flow displacements in porous media can lead to the development of hydrodynamic instabilities in the form of fingerlike structures at the interface between the two fluids. In homogeneous porous media, the instability results from viscosities mismatch and is referred to as viscous fingering and/or densities mismatch where it is known as the RayleighTaylor instability. Both instabilities can be observed in a variety of processes that span a wide range of fields that include chemical, petroleum and environmental sectors. Hence a better understanding of these instabilities is crucial for the design, optimization and improvement of numerous processes encountered, for example, in the operation of packed bed reactors, production and enhanced recovery of oil, decontamination of soil as well as polymer processing.

Extensive studies have been conducted to analyze the effects of different factors on the instability including the viscosity ratio [1], dispersion [2], heat transfer [3], and chemical reaction [4] among others. Extensive reviews on this topic are found in [5] and [6].

The vast majority of existing studies have focused on miscible displacements when dispersion plays an important role in addition to the viscosity/density ratios and the injection rates. However in numerous practical applications, the flow displacements are immiscible and surface tension forces become a major factor in the development and subsequent fate of the instability. In terms of studies dealing with immiscible displacements, one must mention the pioneering work [7] who have analyzed the early development of the instabilities in radial Hele-Shaw cells. Subsequent studies analyzed the effects of the injection flow rate, surface tension and fluids viscosity [8-10].

Moreover due to their inherent complexity, instabilities involving non-Newtonian flows have received very limited attentions whether in the case of miscible or immiscible displacements. One must however mention the studies [11-13] who have examined the dynamics of non-Newtonian flow displacements in Hele-Shaw cells for either miscible or immiscible fluids. The objective of this study is to examine immiscible radial flow displacements that involve nonNewtonian shear thinning fluids. The objective is to understand and relate the flow dynamics to the rheological characteristics of the fluids. Such flow displacements are encountered in a wide variety of process such as resin transfer moulding in the manufacturing of composite materials and enhanced oil recovery using polymer solutions. 


\section{Problem Formulation and Solution}

In this section, the geometry of the flow is presented, followed by a presentation of the governing equations and boundary conditions. Finally, the numerical technique to track the interface between the two phases, is briefly described.

\subsection{Flow Geometry}

The domain in which the flow evolves consists of a two-dimensional homogeneous porous medium with constant permeability $\mathrm{K}$, where the flow develops radially from a centre source. A fluid of viscosity $\mu_{1}$ is injected in the centre of the domain to displace radially a second fluid of viscosity $\mu_{2}$, that initially occupies the whole medium. It is assumed that both fluids are fully immiscible with a surface tension $\sigma$, and that the displacement is isothermal. In this study, the displaced fluid is assumed to be non-Newtonian with the viscosity depending on the shear rate; $\mu_{2}(\dot{\gamma})$. A related variable that will be used throughout this study is the mobility $\beta_{i}=K / \mu_{i}, i=1,2$ defined as the ratio of the medium permeability and the fluid viscosity. A schematic of the flow is depicted in Figure 1.

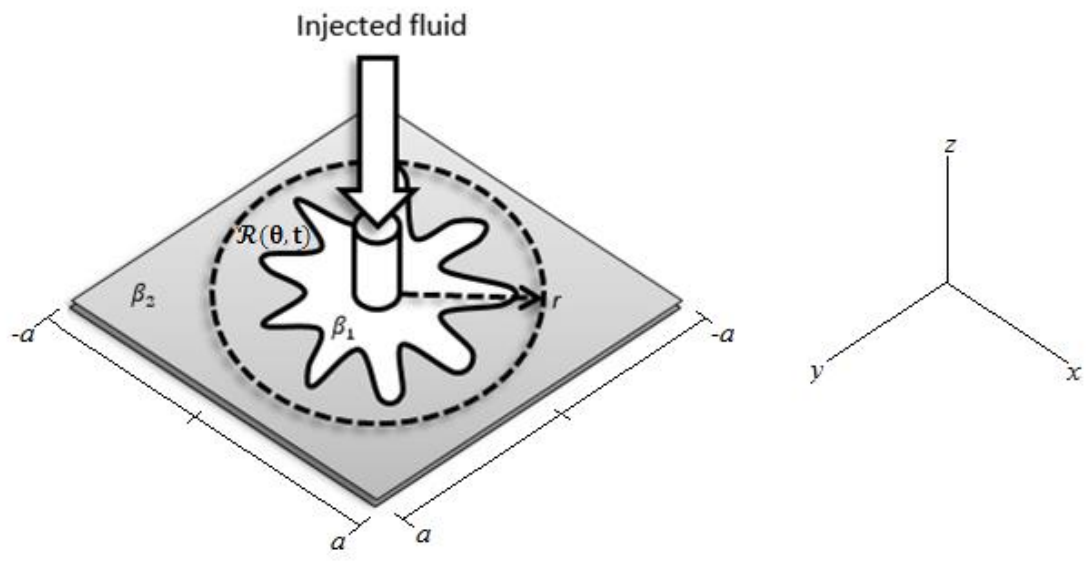

Fig. 1: Schematic of the flow.

\subsection{Governing Equations}

The mathematical model describing the immiscible displacement consists of the continuity equation (mass conservation) and Darcy's law (momentum conservation) [10]:

$$
\begin{gathered}
\nabla . \mathrm{U}= \begin{cases}\phi, & r \leq R_{0} \\
0, & r>R_{0}\end{cases} \\
\nabla p= \begin{cases}-\frac{1}{\beta_{1}} U & \text { fluid } 1 \\
-\frac{1}{\beta_{2}(\dot{\gamma})} U & \text { fluid } 2\end{cases}
\end{gathered}
$$

In the above model, the equations are formulated in each region occupied by either fluid 1 or fluid 2 . The continuity equation involves a source injection rate $\phi$ in a region of the domain, determined by a radius $R_{0}$, which also corresponds to the initial radius of the interface. Darcy's law expresses the proportionality between the fluid velocity $(U)$ and the pressure gradient through the mobility $\left(\beta_{i}\right)$. The interface between the two fluids at any time $(t)$ and angle $(\theta)$ is $R(\theta, t)$. In this study, the displaced fluid; fluid 2 is assumed to be non-Newtonian with a viscosity governed by the Carreau rheological model:

$$
\mu_{2}=\mu_{20}\left[1+(\lambda \dot{\gamma})^{2}\right]^{\frac{n-1}{2}}
$$


where $\dot{\gamma}$ is the magnitude of the rate-of-strain tensor, $\mu_{20}$ is the zero-shear viscosity and $n$ is the power law index. The boundary conditions at the interface are:

$$
[p]_{1}^{2}=\sigma \kappa, \quad\left[U_{n}\right]_{1}^{2}=0
$$

where the brackets refer to the difference of values at the interface between the two fluids. In the above equations, $\kappa$ is the curvature of the interface while $\sigma$ is the surface tension. The governing equation are made dimensionless using $R_{0}$, $\frac{1}{\phi_{0}}, \phi_{0} R_{0}$ and $\frac{\phi_{0} R_{0}{ }^{2}}{\beta_{1}}$ as reference length, time, velocity and stress; where $Q_{0}=\pi R_{0}{ }^{2} \phi_{0}$ is the injection volumetric rate per unit length. The resulting dimensionless equations and boundary conditions are characterized by four major dimensionless groups, the mobility ratio $\mathrm{M}=\frac{\beta_{1}}{\beta_{20}}$, the dimensionless surface tension $\sigma^{*}=\frac{\sigma \beta_{1}}{R_{0}{ }^{3} \phi_{0}}$ which can be regarded as an inverse Capillary number, the Deborah number $D e=\lambda \phi_{0}$ and the power law index $n$.

\subsection{Numerical Technique}

The governing equations are solved using a hybrid method introduced by [14] based on a combination of the Immersed Interface Method (IIM) and the Level Set Method (LSM). IIM consists of a finite difference method to solve Eqs. (1) - (2) by implementing correction terms at the interface to account for the jump conditions, Eq. (4). The conditions at the boundaries of the domain consist of the pressure resulting from the radial outflow [14]. More details about the technique, validation of its numerical convergence and comparisons with experimental measurements are found in [10].

\section{Results}

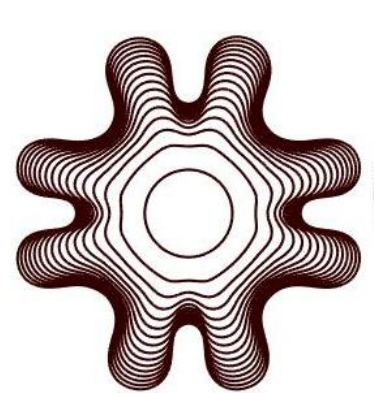

(a)

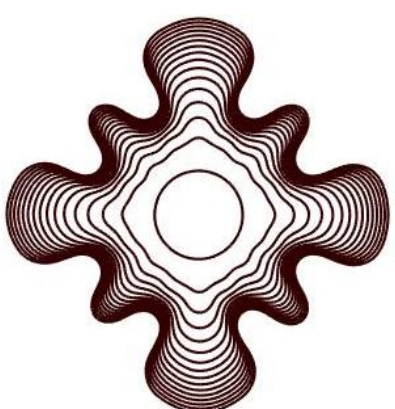

(b)

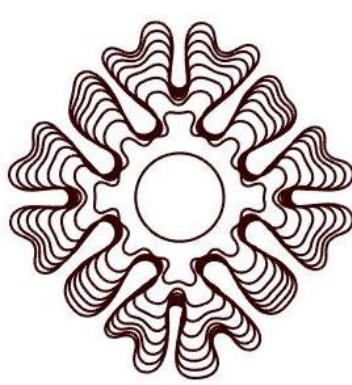

(c)

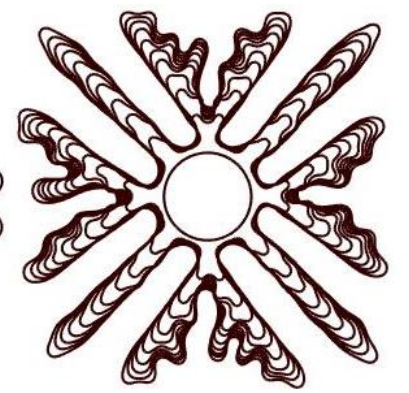

(d)

Fig. 2: Time sequences of interfacial contours for (a) $D e=0.00$, (b) $D e=0.15$, (c) $D e=0.30$ and (d) $D e=0.60 . M=100, \sigma^{*}=1.5, n=0.9$.

Figure 2 depicts interfacial contours for the Newtonian flow displacement $(D e=0.0)$ and the one involving a shear thinning fluid of power law index $n=0.9$ and for three different values of the Deborah number. Results are presented for $M=110$ and $\mathrm{Ca}=66$ and each frame shows a sequence of the interface as it progresses with time. In the Newtonian case, the interfaces is slowly distorted leading ultimately to the development of eight distinct smooth fingers. The structure of the interfaces is strongly changed when the displaced fluid is shear thinning, and the larger the Deborah number, the more complex the interface which can exhibit secondary instabilities in the form of tip-splitting (see figure 2-c) and sidebranching (figure 2-d). It should be noted that the largest $D e$, the fingers are thinner and tend to extend further towards the domain boundary with less inter-finger interactions. 


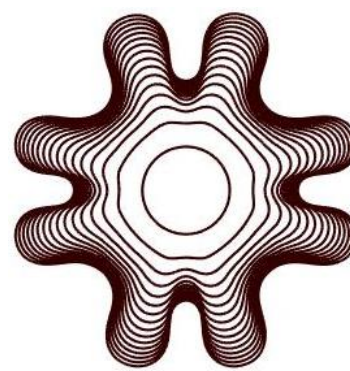

(a)

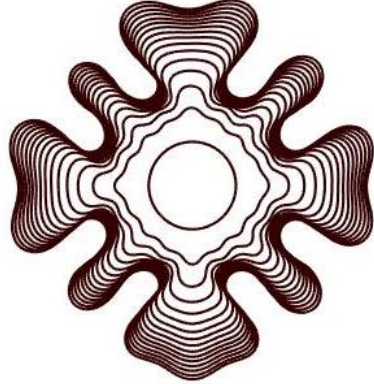

(b)

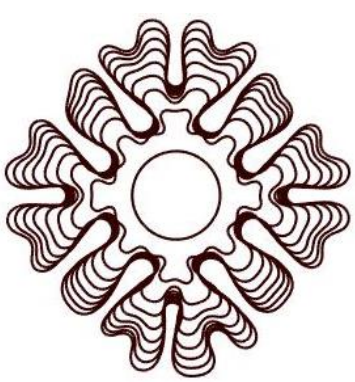

(c)

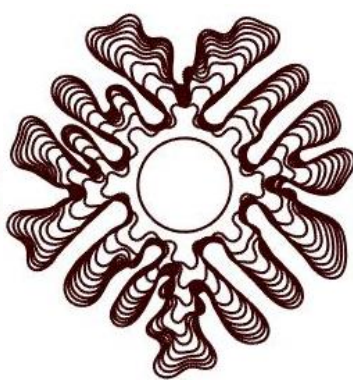

(d)

Fig. 3: Time sequences of interfacial contours for (a) $n=1.00$, (b) $n=0.95$, (c) $n=0.90$ and (d) $n=0.85 . M=100, \sigma^{*}=1.5, D e=0.3$.

The effects of the power law index $n$ on the interfacial instability are presented in fig. 3. It should be noted that the problems reduces to the Newtonian case for $n=1$, and the smaller the values of $n$ the stronger the shear thinning effects. It is clear that the more shear-thinning the displaced fluid is as exemplified by smaller $n$, the more complex the interfacial structures. However even though the fingers are more complex than for large $D e$, they tend comparatively to extend less downstream.

\section{Conclusions}

In this study, the effects of non-Newtonian rheological behaviour on fingering instabilities in homogeneous porous media were examined. The analysis focuses on the development of immiscible displacements where the displacing fluid is Newtonian and the displaced one shear thinning governed by Carreau's model. The development and progress of the instabilities are illustrated through time-contours of the interface. It is found that stronger shear thinning effects resulting from larger Deborah number $D e$ or smaller power law index $n$, lead to more complex finger structures. The effects of $n$ were however found to be more important. This result can be explained by the fact that smaller values $n$ imply a stronger rate of decrease of the viscosity with shear rate, which is expected to induce larger local mobility ratios at the tip of the fingers that tend to extend far downstream. This may explain the reported complex structures. Larger $D e$ translate in the effects of shear thinning appearing at smaller shear rates and extend over a wide range for larger $n$.

\section{Acknowledgements}

The authors wish to acknowledge financial support from the Natural Sciences and Engineering Research Council of Canada (NSERC). The use of computational resources from WestGrid is gratefully acknowledged.

\section{References}

[1] C. T. Tan, and G. M. Homsy, "Simulation of nonlinear viscous fingering in miscible displacement: Rectilinear flow," Physics of Fluids, vol. 29, pp. 3549-3556, 1986.

[2] K. Ghesmat and J. Azaiez, "Viscous fingering instability in porous media: Effect of anisotropic velocity dependent dispersion tensor," Transport in Porous Media, vol. 73, no. 3, pp. 297-318, 2009.

[3] X. Kong, M. Haghighi, Y. C. Yortsos, "Visualization of steam displacement of heavy oils in a Hele-Shaw cell," Fuel, vol. 71, pp. 1465-1471, 1992.

[4] S. H. Hejazi and J. Azaiez, "Hydrodynamic instability in the transport of miscible reactive slices through porous media," Physical Review E, vol. 81, p. 056321, 2010.

[5] G. M. Homsy, "Viscous fingering in porous media," Annual Review of Fluid Mechanics, vol. 19, pp. 271-311, 1987.

[6] K. V. McCloud and J. V. Maher, "Experimental perturbations to Saffman-Taylor flow," Physics Reports, vol. 260, pp. 139-185, 1995.

[7] L Paterson, "Radial Fingering in a Hele-Shaw Cell," Journal of Fluid Mechanics, vol. 113, pp. 513-529, 1981.

[8] J.-D. Chen, "Growth of radial viscous fingers in a Hele-Shaw cell," Journal of Fluid Mechanics, vol. 201, pp. 223242, 1989. 
[9] S. S. Cardoso and A. W. Woods, "The formation of drops through viscous instability," Journal of Fluid Mechanics, vol. 289, pp. 351-378, 1995.

[10] T. F. Lins and J. Azaiez, "Resonance-like dynamics in radial cyclic injection flows of immiscible fluids in homogeneous porous media," Journal of Fluid Mechanics, vol. 819, pp. 713-729, 2017.

[11] M. Kawaguchi, K. Makino and T. Kato, "Viscous fingering patterns in polymer solutions," Physica D, vol. 109, pp. 325-332, 1997.

[12] L. Kondic, M. J. Shelley and P. Palffy-Muhoray, "Non-Newtonian Hele-Shaw flow and the Saffman-Taylor instability," Physical Review Letters, vol. 80, pp. 1433-1436, 1998.

[13] J. Azaiez and B. Singh, "Stability of Miscible Displacements of Shear Thinning Fluids in a Hele-Shaw Cell," Physics Fluids vol. 14, pp. 1557-1571, 2002.

[14] T. Y. Hou, Z. Li, S. Osher and H. Zhao, "A hybrid method for moving interface problems with application to the Hele-Shaw flow," Journal of Computational Physics, vol. 134, no. 2, pp. 236-252, 1997. 